\title{
Efficient Interference Mitigating Strategies for Two-Way Relay Channels
}

\author{
Agisilaos Papadogiannis \\ Department of Signals and Systems \\ Chalmers University of Technology \\ SE-412 96, Göteborg, Sweden \\ agisilaos.papadogiannis@chalmers.se
}

\author{
Alister G. Burr \\ Department of Electronics \\ University of York \\ YO10 5DD, York, UK \\ agb1@ohm. york.ac.uk
}

\author{
Meixia Tao \\ Department of Electronic Engineering \\ Shanghai Jiao Tong University \\ Shanghai 200240, China \\ mxtaoesjtu.edu.cn
}

\begin{abstract}
Hierarchical wireless networks have the potential to provide the needed capacity to a large number of mobile stations (MSs). According to this system architecture, the MSs are served by a dense network of fixed relay nodes (RNs) fed by a small number of large hub base stations (HBSs). In such deployment, high spectral efficiencies can be achieved if the RNs act as twoway relays; however this gives rise to co-channel interference (CCI) which needs to be mitigated. In order to gain insights on the impact of CCI to this scenario we consider an HBS with two highly directional antennas communicating with two MSs via two interfering two-way RNs. We investigate the average maximum sum-rate of two cooperative strategies. The first is based on Decode-and-Forward (DF) and network coding and acts as a baseline. The second strategy is based on Amplifyand-Forward (AF) and Network MIMO processing over both the backhaul and access channels. Furthermore we devise some cooperative protocols that utilize two, three or four time slots. It is shown that the 2-slot schemes perform generally better and that the DF strategy achieves superior performance when CCI is low while AF with Network MIMO is superior when CCI is high.
\end{abstract}

Index Terms-Two-way relaying, hierarchical networks, decode-and-forward (DF), amplify-and-forward (AF), Network MIMO, network coding.

\section{INTRODUCTION}

The use of relay nodes (RNs) has been recognized as a very promising avenue towards future wireless communications [1], [2]. RN-enabled communications are seen as cost-effective means to improve connectivity, transmission reliability and quality-of-service without requiring a large number of antenna elements per network node [1]-[3]. RNs can be inexpensive fixed wireless nodes or even user terminals that relay signals intended for other users [4], [5]. Recently it has been identified that RN-enabled networks, apart from achieving the aforementioned gains, can also greatly increase the achievable capacity density of the network, measured in bits $/ \mathrm{sec} / \mathrm{km}^{2}$ [6], [7].

A promising hierarchical architecture for future wireless systems entails that mobile stations (MSs) are served in a twohop fashion via a dense grid of fixed RNs deployed at the street level. The RNs are fed by a small number of hub base stations (HBSs) deployed above rooftops [6], [7]. The spectral efficiency of such systems can be improved with the use of full-duplex RNs, i.e., RNs that can transmit and receive at the same time and frequency. However this type of RNs is hard to implement [8]. Alternatively, spectral efficiency can be enhanced using two-way relaying with half-duplex RNs [8]-[12]. Although such technique is promising, the dense RN deployment of hierarchical networks results in high co-channel interference (CCI) which limits system performance. In the literature the issue of CCI has been identified for two-way relaying systems [12], [13], however its effects have not been investigated for the hierarchical system architecture of interest.

In this paper we investigate how CCI impacts on hierarchical networks and propose cooperative strategies that mitigate CCI. We study the average maximum sum rate (AMSR) performance of a hierarchical network consisting of an HBS with two highly directional antennas, two interfering two-way RNs and two MSs. We propose two general cooperative strategies; the first is based on Decode-and-Forward (DF) with the aid of network coding and serves as our baseline. The second is based on Amplify-and-Forward (AF) combined with Network MIMO processing applied over both the backhaul and the access networks. Furthermore we devise some communication protocols requiring two, three or four time slots that are combined with our proposed strategies. We show that for the considered system scenario the 2-slot protocols perform best. The DF-based strategy performs better in the low CCI regime while the AF-based strategy exploits CCI in the uplink and greatly outperforms DF when CCI is dominant.

The remainder of this paper is structured as follows. In Section II the signal and system model is presented. In Sections III and IV the proposed schemes based on DF relaying combined with network coding and AF relaying coupled with Network MIMO are presented and discussed. In Section V simulation results are shown and in Section VI the paper is concluded.

Notations: Vectors and matrices are denoted by boldface lowercase letters and boldface capital letters respectively. $\mathbf{A}[i, j]$ represents the $i j$-th element of a matrix. The transpose, transpose conjugate, the inverse and the pseudo-inverse of a matrix $\mathbf{A}$ are denoted by $\mathbf{A}^{T}, \mathbf{A}^{H}, \mathbf{A}^{-1}$ and $\mathbf{A}^{\dagger}$ respectively. The XOR operation is denoted by $\oplus$. Furthermore $\mathbb{E}[$.] denotes expectation and $C(x) \triangleq \log _{2}(1+x)$.

\section{Signal AND System Model}

We consider a hierarchical system consisting of two singleantenna MSs (nodes 1 and 2), two half-duplex single-antenna 


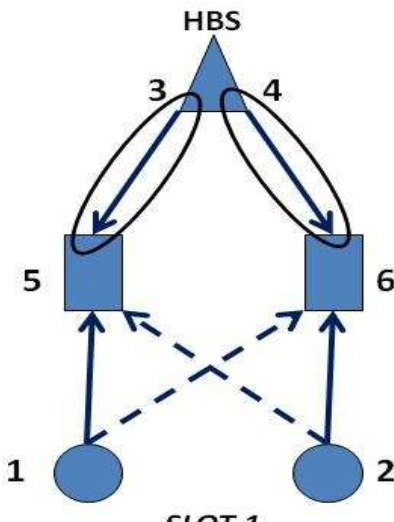

SLOT 1

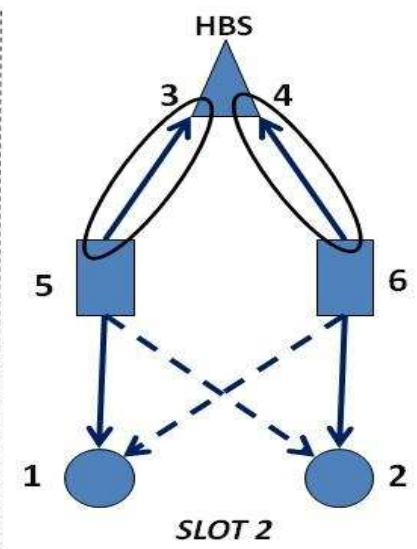

SLOT 2
Fig. 1. The considered system scenario under a 2-slot protocol: an HBS with two directional antennas (nodes 3,4 ), two MSs (nodes 1, 2), and two RNs (nodes 5, 6).

RNs (nodes 5 and 6), and an HBS with two directional antennas which are assumed to create two non-interfering channels (designated as nodes 3 and 4) as shown in Fig. 1. The MS and RN antennas are assumed to be omni-directional. The HBS and the MS nodes want to exchange messages via the RN nodes; node 1 with node 3 and node 2 with node 4 . Nodes 1 and 2 receive/cause interference from/to nodes 6 and 5 respectively. The wireless links between the HBS antennas and the RNs are defined as the backhaul network, while the links between the RNs and the MSs are defined as the access network. The wireless channels between any pair of nodes are assumed to experience flat fading. The channel coefficient between nodes $k$ and $n$ is

$$
h_{k, n}=\Gamma_{k, n} \sqrt{\bar{\gamma}_{k, n}}
$$

where $\Gamma_{k, n}$ denotes the normalized fading coefficient and $\bar{\gamma}_{k, n}$ denotes the average signal-to-noise ratio (SNR) of the link. Transmission is corrupted by unit variance zero-mean circularly symmetric additive white Gaussian noise (AWGN).

The MS and HBS nodes are assumed to be transmitting unit variance symbols grouped in vector $\mathbf{x}=\left[x_{1}, x_{2}, x_{3}, x_{4}\right]^{T}$. The RN nodes receive the signal vector $\mathbf{y}_{R}=\left[y_{5}, y_{6}\right]^{T}$. It should be noted that the elements of vectors $\mathbf{x}$ and $\mathbf{y}_{R}$ can be transmitted and received in different time slots depending on the employed cooperative protocol.

\section{DF WITH NETWORK CODING}

In this section we present a cooperative strategy and some communication protocols based on DF. The RNs decode the wanted signals treating the received interference as noise. The decoded symbols are combined with the use of the bitwise XOR operation and forwarded to the destination nodes. Communication can take place in two, three or four time slots. As the number of time slots grows, the impact of CCI on the attained performance becomes less significant. However increasing the number of slots incurs a pre-log penalty that limits the achievable capacity.

\section{A. 2-slot DF-XOR}

In the first time slot the HBS transmits the symbol vector $\mathbf{x}_{B}=\left[x_{3}, x_{4}\right]^{T}$ and the MSs transmit the symbol vector $\mathbf{x}_{U}=$ $\left[x_{1}, x_{2}\right]^{T}$. Node 5 decodes symbols $x_{1}, x_{3}$ treating $h_{5,2} x_{2}$ as noise and node 6 decodes $x_{2}, x_{4}$ treating $h_{6,1} x_{1}$ as noise. In the second time slot nodes 5 and 6 transmit $x_{5}=x_{1} \oplus x_{3}$ and $x_{6}=x_{2} \oplus x_{4}$ respectively. Nodes 1 and 3 decode $x_{5}$ and retrieve the symbol $x_{3}$ and $x_{1}$ respectively. Similarly, nodes 2 and 4 decode $x_{6}$ and retrieve $x_{4}$ and $x_{2}$ respectively.

We define some rate expressions for the multiple access (MAC) phase of the first time slot in the following, which will be used as rate constraints later. $C_{15}=\frac{1}{2} C\left(\frac{\left|h_{5,1}\right|^{2}}{\left|h_{5,2}\right|^{2}+1}\right)$, $C_{35}=\frac{1}{2} C\left(\frac{\left|h_{5,3}\right|^{2}}{\left|h_{5,2}\right|^{2}+1}\right), C_{M 5}=\frac{1}{2} C\left(\frac{\left|h_{5,1}\right|^{2}+\left|h_{5,3}\right|^{2}}{\left|h_{5,2}\right|^{2}+1}\right)$, $C_{26}=\frac{1}{2} C\left(\frac{\left|h_{6,2}\right|^{2}}{\left|h_{6,1}\right|^{2}+1}\right), C_{46}=\frac{1}{2} C\left(\frac{\left|h_{6,4}\right|^{2}}{\left|h_{6,1}\right|^{2}+1}\right)$ and $C_{M 6}=$ $\frac{1}{2} C\left(\frac{\left|h_{6,2}\right|^{2}+\left|h_{6,4}\right|^{2}}{\left|h_{6,1}\right|^{2}+1}\right)$. The rate expressions for the broadcast (BC) phase of the second time slot are defined as: $C_{53}=$ $\frac{1}{2} C\left(\left|h_{3,5}\right|^{2}\right), C_{51}=\frac{1}{2} C\left(\frac{\left|h_{1,5}\right|^{2}}{\left|h_{1,6}\right|^{2}+1}\right), C_{62}=\frac{1}{2} C\left(\frac{\left|h_{2,6}\right|^{2}}{\left|h_{2,5}\right|^{2}+1}\right)$ and $C_{64}=\frac{1}{2} C\left(\left|h_{4,6}\right|^{2}\right)$.

\section{B. 3-slot DF-XOR}

According to this protocol, in the first time slot nodes 1 and 3 transmit symbols $x_{1}$ and $x_{3}$ respectively and RN 5 decodes them in the absence of CCI. In the second time slot nodes 2 and 4 transmit symbols $x_{2}$ and $x_{4}$ respectively and RN 6 decodes them also in the absence of CCI. In the third time slot nodes 5 and 6 transmit $x_{5}=x_{1} \oplus x_{3}$ and $x_{6}=x_{2} \oplus$ $x_{4}$ respectively. Furthermore, nodes 1 and 3 decode $x_{5}$ and retrieve the symbol $x_{3}$ and $x_{1}$ respectively. Similarly, nodes 2 and 4 decode $x_{6}$ and retrieve $x_{4}$ and $x_{2}$ respectively. It should be noted that nodes 1 and 2 treat $h_{1,6} x_{6}$ and $h_{2,5} x_{5}$ as noise while decoding $x_{3}$ and $x_{4}$.

The rate expressions for the MAC phase of the 3slot protocol are the following: $C_{15}=\frac{1}{3} C\left(\left|h_{5,1}\right|^{2}\right)$, $C_{35}=\frac{1}{3} C\left(\left|h_{5,3}\right|^{2}\right), C_{M 5}=\frac{1}{3} C\left(\left|h_{5,1}\right|^{2}+\left|h_{5,3}\right|^{2}\right)$, $C_{26}=\frac{1}{3} C\left(\left|h_{6,2}\right|^{2}\right), C_{46}=\frac{1}{3} C\left(\left|h_{6,4}\right|^{2}\right)$ and $C_{M 6}=$ $\frac{1}{3} C\left(\left|h_{6,2}\right|^{2}+\left|h_{6,4}\right|^{2}\right)$. The rate expressions for the BC phase are defined as: $C_{53}=\frac{1}{3} C\left(\left|h_{3,5}\right|^{2}\right), C_{51}=\frac{1}{3} C\left(\frac{\left|h_{1,5}\right|^{2}}{\left|h_{1,6}\right|^{2}+1}\right)$, $C_{62}=\frac{1}{3} C\left(\frac{\left|h_{2,6}\right|^{2}}{\left|h_{2,5}\right|^{2}+1}\right)$ and $C_{64}=\frac{1}{3} C\left(\left|h_{4,6}\right|^{2}\right)$.

\section{4-slot DF-XOR}

The 4-slot protocol frees the system from CCI and serves as a performance benchmark. In the first time slot nodes 1 and 3 transmit symbols $x_{1}$ and $x_{3}$ respectively and RN 5 decodes them. In the second time slot node 5 transmits $x_{5}=x_{1} \oplus x_{3}$ and nodes 1 and 3 decode $x_{5}$ and retrieve symbols $x_{3}$ and $x_{1}$ respectively. Similarly, in the third time slot nodes 2 and 4 transmit symbols $x_{2}$ and $x_{4}$ respectively and RN 6 decodes them. In the fourth time slot node 6 transmits $x_{6}=x_{2} \oplus x_{4}$ and nodes 2 and 4 decode $x_{6}$ and retrieve symbols $x_{2}$ and $x_{4}$ respectively 
The rate expressions for the MAC phase of the 4slot protocol are the following: $C_{15}=\frac{1}{4} C\left(\left|h_{5,1}\right|^{2}\right)$, $C_{35}=\frac{1}{4} C\left(\left|h_{5,3}\right|^{2}\right), \quad C_{M 5}=\frac{1}{4} C\left(\left|h_{5,1}\right|^{2}+\left|h_{5,3}\right|^{2}\right)$, $C_{26}=\frac{1}{4} C\left(\left|h_{6,2}\right|^{2}\right), C_{46}=\frac{1}{4} C\left(\left|h_{6,4}\right|^{2}\right)$ and $C_{M 6}=$ $\frac{1}{4} C\left(\left|h_{6,2}\right|^{2}+\left|h_{6,4}\right|^{2}\right)$. The rate expressions for the BC phase are defined as: $C_{53}=\frac{1}{4} C\left(\left|h_{3,5}\right|^{2}\right), C_{51}=\frac{1}{4} C\left(\left|h_{1,5}\right|^{2}\right)$, $C_{62}=\frac{1}{4} C\left(\left|h_{2,6}\right|^{2}\right)$ and $C_{64}=\frac{1}{4} C\left(\left|h_{4,6}\right|^{2}\right)$.

Let $\mathbf{r}=\left[R_{1}, R_{3}, R_{2}, R_{4}\right]^{T}$ be the vector containing the transmit rates of HBS and MS nodes. Let $\mathbf{b}_{1}=\left[C_{15}, C_{35}, C_{M 5}, C_{26}, C_{46}, C_{M 6}\right]^{T}, \quad \mathbf{b}_{2}=$ $\left[C_{53}, C_{51}, C_{64}, C_{62}\right]^{T}$ be the vectors containing the rate constraints of the MAC and BC phases respectively. The maximum sum-rate can be expressed as

$$
\begin{aligned}
R_{D F}= & \max _{\mathbf{r}} \sum_{k=1}^{4} R_{k} \\
\text { s.t. } & \mathbf{A} \mathbf{r} \leq \mathbf{b}_{1} \\
& \mathbf{I} \mathbf{r} \leq \mathbf{b}_{2}
\end{aligned}
$$

where $\mathbf{I}$ is the identity matrix and

$$
\mathbf{A}=\left[\begin{array}{llll}
1 & 0 & 0 & 0 \\
0 & 1 & 0 & 0 \\
1 & 1 & 0 & 0 \\
0 & 0 & 1 & 0 \\
0 & 0 & 0 & 1 \\
0 & 0 & 1 & 1
\end{array}\right]
$$

\section{AF WITH NeTWORK MIMO}

In the present section it is proposed that $\mathrm{AF}$ relaying can be applied combined with Network MIMO in order to mitigate the effects of CCI. The proposed strategy can utilize two or three time slots.

\section{A. 2-slot $A F$}

In the first slot MS and HBS nodes transmit their symbols, grouped in vector $\mathbf{x}$, and the RNs receive

$$
\mathbf{y}_{R}=\mathbf{H}_{R} \mathbf{x}+\mathbf{n}_{R}
$$

where $\mathbf{n}_{R}$ is a vector of AWGN coefficients, $\mathbb{E}\left[\mathbf{n}_{R} \mathbf{n}_{R}^{H}\right]=\mathbf{I}$, and

$$
\mathbf{H}_{R}=\left[\begin{array}{cccc}
h_{5,1} & h_{5,2} & h_{5,3} & 0 \\
h_{6,1} & h_{6,2} & 0 & h_{6,4}
\end{array}\right] .
$$

Note that the zero elements in $\mathbf{H}_{R}$ reflect the fact that the HBS antennas (nodes 3 and 4) are assumed not to interfere. In the second time slot both RNs transmit an amplified version of their received signal and the amplification factors take the following values for $\mathrm{RN}$ nodes 5 and 6

$$
\begin{aligned}
& \alpha_{5}=\left[\left|h_{5,1}\right|^{2}+\left|h_{5,2}\right|^{2}+\left|h_{5,3}\right|^{2}+1\right]^{-1 / 2} \\
& \alpha_{6}=\left[\left|h_{6,1}\right|^{2}+\left|h_{6,2}\right|^{2}+\left|h_{6,4}\right|^{2}+1\right]^{-1 / 2} .
\end{aligned}
$$

The MS and the HBS antennas receive the signal vectors $\mathbf{y}_{U}=\left[y_{1}, y_{2}\right]^{T}$ and $\tilde{\mathbf{y}}_{B}=\left[\tilde{y}_{1}, \tilde{y}_{2}\right]^{T}$ respectively, which can be expressed as

$$
\begin{aligned}
\mathbf{y}_{U} & =\mathbf{H}_{U} \mathbf{H}_{R} \mathbf{x}+\mathbf{H}_{U} \mathbf{n}_{R}+\mathbf{n}_{U}=\tilde{\mathbf{H}}_{U} \mathbf{x}+\tilde{\mathbf{n}}_{U} \\
\tilde{\mathbf{y}}_{B} & =\mathbf{H}_{B} \mathbf{H}_{R} \mathbf{x}+\mathbf{H}_{B} \mathbf{n}_{R}+\mathbf{n}_{B}=\tilde{\mathbf{H}}_{B} \mathbf{x}+\tilde{\mathbf{n}}_{B}
\end{aligned}
$$

where $\tilde{\mathbf{H}}_{U}=\mathbf{H}_{U} \mathbf{H}_{R}, \tilde{\mathbf{H}}_{B}=\mathbf{H}_{B} \mathbf{H}_{R}, \tilde{\mathbf{n}}_{U}=\mathbf{H}_{U} \mathbf{n}_{R}+\mathbf{n}_{U}$, $\tilde{\mathbf{n}}_{B}=\mathbf{H}_{B} \mathbf{n}_{R}+\mathbf{n}_{B}$ and

$$
\begin{aligned}
\mathbf{H}_{U} & =\left[\begin{array}{cc}
\alpha_{5} h_{1,5} & \alpha_{6} h_{1,6} \\
\alpha_{5} h_{2,5} & \alpha_{6} h_{2,6}
\end{array}\right] \\
\mathbf{H}_{B} & =\left[\begin{array}{cc}
\alpha_{5} h_{3,5} & 0 \\
0 & \alpha_{6} h_{4,6}
\end{array}\right] .
\end{aligned}
$$

The noise covariances are

$$
\begin{aligned}
& \mathbf{R}_{\tilde{n}_{U}}=\operatorname{diag}\left\{\sum_{n=1}^{2}\left|\mathbf{H}_{U}[1, n]\right|^{2}+1, \sum_{n=1}^{2}\left|\mathbf{H}_{U}[2, n]\right|^{2}+1\right\} \\
& \mathbf{R}_{\tilde{n}_{B}}=\operatorname{diag}\left\{\left|\mathbf{H}_{B}[1,1]\right|^{2}+1,\left|\mathbf{H}_{B}[2,2]\right|^{2}+1\right\} \text {. }
\end{aligned}
$$

As MS nodes are remote they can only process signals individually. Node 1 decodes the message of node 3 and node 2 that of node 4 . For the 2 -slot protocol the achievable rates for the transmission of nodes 3 and 4 are

$$
\begin{aligned}
& R_{3}=\frac{1}{2} C\left(\frac{\left|\tilde{\mathbf{H}}_{U}[1,3]\right|^{2}}{\left|\tilde{\mathbf{H}}_{U}[1,2]\right|^{2}+\left|\tilde{\mathbf{H}}_{U}[1,4]\right|^{2}+\mathbf{R}_{\tilde{n}_{U}}[1,1]}\right) \\
& R_{4}=\frac{1}{2} C\left(\frac{\left|\tilde{\mathbf{H}}_{U}[2,4]\right|^{2}}{\left|\tilde{\mathbf{H}}_{U}[2,1]\right|^{2}+\left|\tilde{\mathbf{H}}_{U}[2,3]\right|^{2}+\mathbf{R}_{\tilde{n}_{U}}[2,2]}\right) .
\end{aligned}
$$

Note that nodes 1 and 2 subtract self-interference $\tilde{\mathbf{H}}_{U}[1,1] x_{1}$ and $\tilde{\mathbf{H}}_{U}[2,4] x_{2}$ respectively. The HBS receives two signals from nodes 3 and 4 containing both $x_{1}$ and $x_{2}$, which are jointly processed. Let $\tilde{\mathbf{H}}_{B}=\left[\tilde{\mathbf{H}}_{B 1} \tilde{\mathbf{H}}_{B 2}\right]$ where

$$
\begin{aligned}
\tilde{\mathbf{H}}_{B 1} & =\left[\begin{array}{cc}
\alpha_{5} h_{3,5} h_{5,1} & \alpha_{5} h_{3,5} h_{5,2} \\
\alpha_{6} h_{4,6} h_{6,1} & \alpha_{6} h_{4,6} h_{6,2}
\end{array}\right] \\
\tilde{\mathbf{H}}_{B 2} & =\left[\begin{array}{cc}
\alpha_{5} h_{3,5} h_{5,3} & 0 \\
0 & \alpha_{6} h_{4,6} h_{6,4}
\end{array}\right] .
\end{aligned}
$$

The sub-matrix $\mathbf{H}_{B 2}$ represents self-interference for nodes 3 and 4 and therefore its effects can be canceled. In consequence only $\mathbf{H}_{B 1}$ affects the achievable rate of nodes 1 and 2 whose signals are jointly decoded by nodes 3 and 4 . We assume that $\mathbf{H}_{B 1}$ is fully known by the HBS. In the case of linear detection a beamforming matrix $\mathbf{W}=\left[\mathbf{w}_{1}, \mathbf{w}_{2}\right]$, which is a function of $\mathbf{H}_{B 1}$ representing the global channel state information (CSI), is designed by the HBS and applied to the received signals. $\mathbf{w}_{1}, \mathbf{w}_{2} \in \mathbb{C}^{2 \times 1}$ denote the beamforming vectors corresponding to the signals transmitted by nodes 1 and 2 respectively. The finally extracted signal can be expressed in vector form as

$$
\mathbf{y}_{B}=\mathbf{W} \tilde{\mathbf{y}}_{B}=\mathbf{W} \tilde{\mathbf{H}}_{B 1} \mathbf{x}_{U}+\mathbf{W} \tilde{\mathbf{n}}_{B}
$$


where $\mathbf{x}_{U}=\left[x_{1}, x_{2}\right]^{T}$. Let $\tilde{\mathbf{H}}_{B 1}=\left[\mathbf{h}_{1}, \mathbf{h}_{2}\right]$ where $\mathbf{h}_{k}$ corresponds to node $k$. The achievable rate for nodes $k=1,2$ is

$$
R_{k}=\frac{1}{2} C\left(\frac{\left|\mathbf{w}_{k}^{T} \mathbf{h}_{k}\right|^{2}}{\left|\mathbf{w}_{k}^{T} \mathbf{h}_{n, n \neq k}\right|^{2}+\left\|\mathbf{w}_{k}^{T}\right\|^{2} \mathbf{R}_{\tilde{n}_{B}}[k, k]}\right)
$$

where factors $\left|\mathbf{w}_{k}^{T} \mathbf{h}_{n, n \neq k}\right|^{2}$ and $\left\|\mathbf{w}_{k}^{T}\right\|^{2} \mathbf{R}_{\tilde{n}_{B}}[k, k]$ correspond to inter-node interference and noise enhancement respectively, which both have a detrimental effect.

We assume that the HBS obtains perfect global CSI (matrix $\left.\mathbf{H}_{B 1}\right)$ and acts as a Network MIMO central unit [5]. The beamforming matrix can be based on Zero-Forcing (ZF), where $\mathbf{W}=\tilde{\mathbf{H}}_{B 1}^{\dagger}$, or the Minimum Mean Square Error (MMSE), where $\mathbf{W}=\left(\tilde{\mathbf{H}}_{B 1}^{H} \tilde{\mathbf{H}}_{B 1}+\mathbf{R}_{B}\right)^{-1} \tilde{\mathbf{H}}_{B 1}$. Detection can be improved further if it is performed in a successive fashion, i.e., the detected symbols are subtracted from the remaining received signal. This frees the signal from some interference components and can enhance the achieved capacity. The composite channel $\tilde{\mathbf{H}}_{B 1}=\left[\mathbf{h}_{1}, \mathbf{h}_{2}\right]$ is ordered so that $\left\|\mathbf{h}_{1}\right\| \leq\left\|\mathbf{h}_{2}\right\|$. The beamforming vector $\mathbf{w}_{k}$ corresponding to node $k$ is the first row of matrix $\mathbf{W}_{k}=\hat{\mathbf{H}}_{k}^{\dagger}$ for ZF and $\mathbf{W}_{k}=$ $\left(\hat{\mathbf{H}}_{k}^{H} \hat{\mathbf{H}}_{k}+\mathbf{R}_{B}\right)^{-1} \hat{\mathbf{H}}_{k}^{H}$ for MMSE, where $\hat{\mathbf{H}}_{k}=\left[\mathbf{h}_{k}, \mathbf{h}_{k+1}\right]^{T}$. With successive interference cancellation (SIC), each node expreriences only interference from nodes with higher index. This results in improved performance compared with linear detection. The achievable sum-rate is

$$
R_{A F}=\sum_{k=1}^{4} R_{k}
$$

\section{B. 3-slot AF}

According to this protocol, in the first time slot MS and HBS nodes transmit their symbols and the received signal by the RNs is given by (4). In the second time slot RN 5 transmits with the amplification factor $\alpha_{5}$ and RN 6 remains silent. In the third time slot RN 6 transmits with the amplification factor $\alpha_{6}$ and RN 5 remains silent. The employed RN amplification factors are given by (6).

The received signals by the MSs and the HBS antennas (vectors $\mathbf{y}_{U}$ and $\tilde{\mathbf{y}}_{B}$ respectively), accumulated in the second and third time slot, are as in (7). It should be noted that $\mathbf{H}_{R}$, $\mathbf{H}_{B}$ are as in (5) and (8) respectively, where $\mathbf{H}_{U}$ is as follows

$$
\mathbf{H}_{U}=\left[\begin{array}{cc}
\alpha_{5} h_{1,5} & 0 \\
0 & \alpha_{6} h_{2,6}
\end{array}\right] .
$$

The noise covariances are

$$
\begin{aligned}
& \mathbf{R}_{\tilde{n}_{U}}=\operatorname{diag}\left\{\left|\mathbf{H}_{U}[1,1]\right|^{2}+1,\left|\mathbf{H}_{U}[2,2]\right|^{2}+1\right\} \\
& \mathbf{R}_{\tilde{n}_{B}}=\operatorname{diag}\left\{\left|\mathbf{H}_{B}[1,1]\right|^{2}+1,\left|\mathbf{H}_{B}[2,2]\right|^{2}+1\right\} .
\end{aligned}
$$

The achievable rates for the transmission of nodes 3 and 4 are

$$
\left.\begin{array}{l}
R_{3}=\frac{1}{3} C\left(\frac{\left|\tilde{\mathbf{H}}_{U}[1,3]\right|^{2}}{R_{4}=\frac{1}{3} C\left(\frac{\left|\tilde{\mathbf{H}}_{U}[1,2]\right|^{2}+\mathbf{R}_{\tilde{n}_{U}}[1,1]}{\left.\tilde{\mathbf{H}}_{U}[2,4]\right|^{2}}\right.}\right) . \\
\left|\tilde{\mathbf{H}}_{U}[2,1]\right|^{2}+\mathbf{R}_{\tilde{n}_{U}}[2,2]
\end{array}\right) .
$$

The HBS receives in the second time slot a signal from node 3 and in the third time slot a signal from node 4 . These signals are jointly processed in the third time slot with the use of Network MIMO techniques as above. Therefore achievable rate for nodes $k=1,2$ is

$$
R_{k}=\frac{1}{3} C\left(\frac{\left|\mathbf{w}_{k}^{T} \mathbf{h}_{k}\right|^{2}}{\left|\mathbf{w}_{k}^{T} \mathbf{h}_{n, n \neq k}\right|^{2}+\left\|\mathbf{w}_{k}^{T}\right\|^{2} \mathbf{R}_{\tilde{n}_{B}}[k, k]}\right) .
$$

The achievable rates can be enhanced with the application of SIC techniques as described above.

\section{Numerical RESUlts}

For simplicity, we assume a symmetric interfering two-way relay channel; the wireless links of the access network, the backhaul network and the interfering links experience the same average SNR, i.e., $\bar{\gamma}_{1,5}=\bar{\gamma}_{5,1}=\bar{\gamma}_{2,6}=\bar{\gamma}_{6,2}=\bar{\gamma}_{A C}, \bar{\gamma}_{5,3}=$ $\bar{\gamma}_{3,5}=\bar{\gamma}_{4,6}=\bar{\gamma}_{6,4}=\bar{\gamma}_{B H}$, and $\bar{\gamma}_{5,2}=\bar{\gamma}_{2,5}=\bar{\gamma}_{1,6}=\bar{\gamma}_{6,1}=$ $\bar{\gamma}_{I}$.

Fig. 2 plots the total AMSR versus the average $\operatorname{SNR} \bar{\gamma}_{I}$ for the considered schemes when backhaul and access networks experience identical average SNR $\bar{\gamma}_{B H}=\bar{\gamma}_{A C}=10 \mathrm{~dB}$ and Rayleigh fading, i.e., $\Gamma_{k, n} \sim \mathcal{N C}(0,1)$. As can be seen from Fig. 2, in the low CCI regime $\left(\bar{\gamma}_{I}<0 \mathrm{~dB}\right)$ the 2-slot 2S-DFXOR approach performs better. When CCI becomes dominant, the 2-slot AF scheme performs better as it effectively exploits interference through Network MIMO processing over both the backhaul and access networks. Amongst the AF schemes, the ones taking advantage of SIC are superior. Although the 3slot schemes avoid some interference, they perform worse than those based on 2-slots as the pre-log penalty of $\frac{1}{3}$ is dominant in the considered scenario. The performance of the 4-slot 4S-DF-XOR scheme is not affected by CCI and serves as a baseline. Fig. 3 plots the AMSR for the downlink (DL) and uplink (UL) separately, for the 2-slot and 3-slot DF-XOR and the 2-slot 2S-AF-LMMSE scheme. UL rate is generally higher as the directional HBS antennas eliminate CCI in the second time slot. The AMSR deteriorates when CCI becomes stronger for all cases apart from the UL of the AF scheme. The UL AMSR of AF improves as $\bar{\gamma}_{I}$ increases because the HBS jointly processes the received signals by nodes 3 and 4 using Network MIMO techniques (CCI turns to an advantage).

Fig. 4 plots the total AMSR for the same schemes when the average SNR of the backhaul network is $\bar{\gamma}_{B H}=20 \mathrm{~dB}$ and that of the access network is $\bar{\gamma}_{B H}=10 \mathrm{~dB}$. This is justified by the fact that the backhaul network can be planned to have links of high quality as the positions of the RNs relative to the HBS can be selected appropriately. As expected, the performance of all schemes improves when the quality of backhaul links increases. Furthermore the AF protocols perform relatively better than the case of Fig. 2 . 


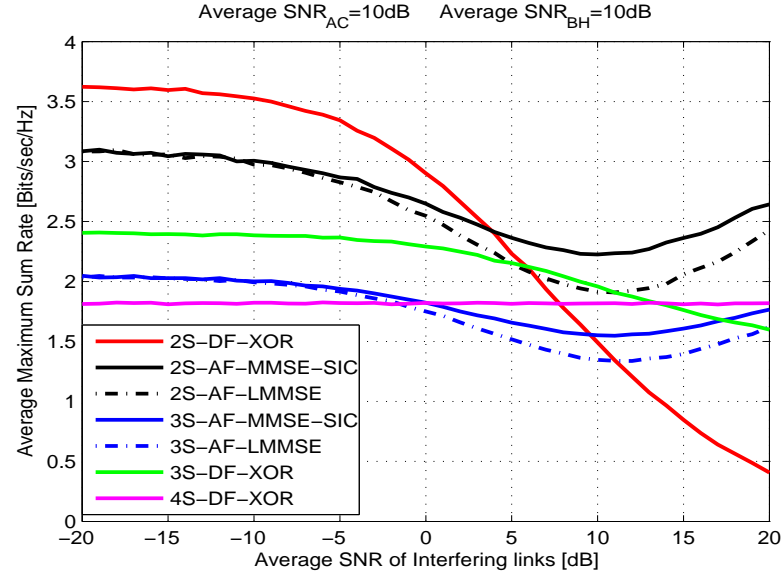

Fig. 2. The total AMSR versus $\bar{\gamma}_{I}$ when $\bar{\gamma}_{B H}=\bar{\gamma}_{A C}=10 \mathrm{~dB}$

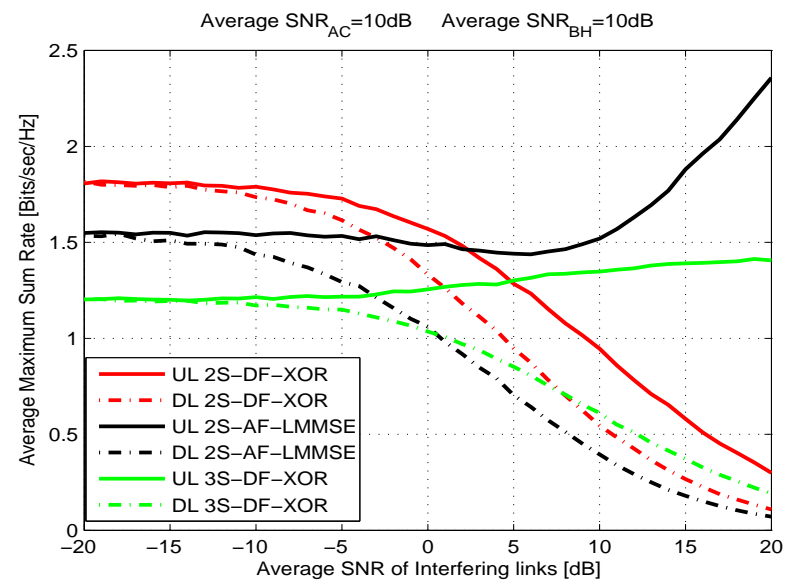

Fig. 3. The AMSR of the UL and DL of 2S-DF-XOR, 3S-DF-XOR and 2S-AF-LMMSE versus $\bar{\gamma}_{I}$ when $\bar{\gamma}_{B H}=\bar{\gamma}_{A C}=10 \mathrm{~dB}$.

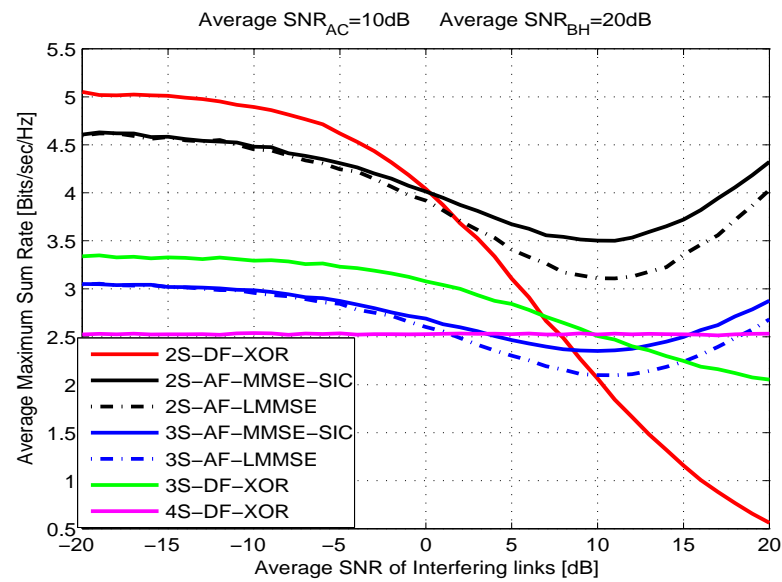

Fig. 4. The total AMSR versus $\bar{\gamma}_{I}$ when $\bar{\gamma}_{B H}=20 \mathrm{~dB}$ and $\bar{\gamma}_{A C}=10 \mathrm{~dB}$.

\section{CONCLUSions}

In this paper we proposed ways of mitigating CCI in a simple dual-hop hierarchical network consisting of an HBS with two directional antennas, two RNs and two MSs. We considered two general cooperative strategies, one based on DF with network coding and another based on AF with Network MIMO. We devised a number of cooperative protocols based on 2, 3 or 4 time slots and compared their performance as a function of the interference strength. It was shown that the 2-slot protocols perform generally better than the 3-slot and 4-slot ones. The DF-XOR scheme achieves superior performance when CCI is weak while the AF with Network MIMO performs better in the high CCI as it turns CCI into an advantage. This is due to the fact that Network MIMO essentially exploits interference at the cost of requiring accurate CSI at the HBS.

\section{ACKNOWLEDGMENTS}

This work was supported by the Swedish Agency for Innovation Systems (VINNOVA), the European Commission's seventh framework programme (FP7/2007-2013) under grant agreement $n^{\circ} 248267$ (BuNGee) and the RCUK under the project "UK-China Science Bridges: R\&D on (B)4G Wireless Mobile Communications" (UC4G) with grant reference EP/G042713/1. The work by M. Tao was supported by the National Natural Science Foundation of China under grant 60902019 and the Shanghai Pujiang Talent Program under grant 09PJ1406000.

\section{REFERENCES}

[1] J. N. Laneman et al., "Cooperative diversity in wireless networks: Efficient protocols and outage behavior," IEEE Trans. on Info. Theory, vol. 50, no. 12, pp. 3062-3080, Dec. 2004.

[2] A. Papadogiannis et al., "A novel framework for the utilisation of dynamic relays in cellular networks," in IEEE ASILOMAR 2008, Pacific Grove, USA, Oct. 2008, pp. 975-979.

[3] R. U. Nabar et al., "Fading relay channels: performance limits and spacetime signal design," IEEE J. on Sel. Areas in Commun., vol. 22, no. 6, pp. 1099-1109, Aug. 2004.

[4] A. Papadogiannis et al., "Bringing mobile relays for wireless access networks into practice- learning when to relay," submitted to IET Communications, 2011.

[5] A. Papadogiannis, "Systems and techniques for Multicell-MIMO and cooperative relaying in wireless networks," Ph.D. dissertation, TELECOM ParisTech, Paris, France, Dec. 2009.

[6] Z. Roth et al., "Vision and architecture supporting wireless Gbit $/ \mathrm{sec} / \mathrm{km}^{2}$ capacity density deployments," in Proc. Future Networks and Mobile Summit (FUNEMS 2010), Florence, Italy, June 2010.

[7] A. Papadogiannis and A. G. Burr, "Multi-beam assisted MIMO - A novel approach to fixed beamforming," in Proc. Future Networks and Mobile Summit (FUNEMS 2011), Warsaw, Poland, June 2011.

[8] B. Rankov and A. Wittneben, "Spectral efficient signaling for halfduplex relay channels," in IEEE ASILOMAR 2005, Pacific Grove, USA, Nov 2005, pp. 1066 - 1071

[9] J. Liu, M. Tao, and Y. Xu, "Rate regions of a two-way Gaussian relay channel," in Proc. IEEE CHINACOM 2009, Xi' an, China, August 2009.

[10] F. Negro, I. Ghauri, and D. Slock, "Maximum weighted sum rate multiuser MIMO amplify-and-forward for two-phase two-way relaying," in Proc. IEEE PIMRC 2010, Istanbul,Turkey, Sept. 2010.

[11] E. Yilmaz et al., "Multi-pair two-way relay channel with multiple antenna relay station," in Proc. ICC 2010, Cape Town, South Africa, May 2010.

[12] I. Maric, R. Dabora, and A. Goldsmith, "On the capacity of the interference channel with a relay," in Proc. ISIT 2008, Toronto, Canada, july 2008, pp. 554-558.

[13] R. Knopp, "Two-way radio networks with a star topology," in International Zurich Seminar on Communications (IZS 2006), Zurich, Switzerland, march 2006, pp. $154-157$. 